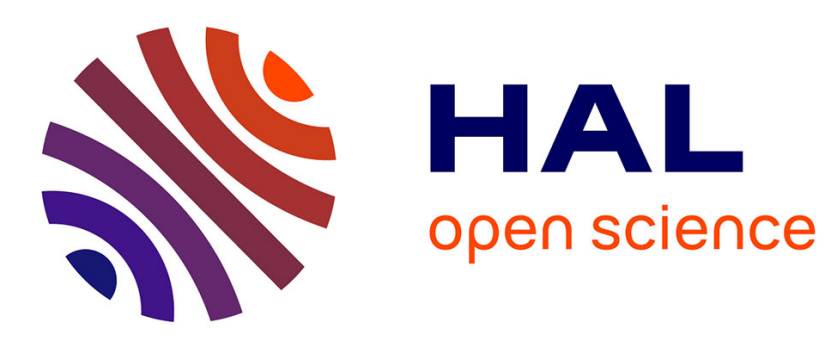

\title{
Gaussian Half-Duplex Relay Channels: Generalized Degrees of Freedom and Constant Gap Result
}

Martina Cardone, Daniela Tuninetti, Raymond Knopp, Umer Salim

\section{To cite this version:}

Martina Cardone, Daniela Tuninetti, Raymond Knopp, Umer Salim. Gaussian Half-Duplex Relay Channels: Generalized Degrees of Freedom and Constant Gap Result. IEEE International Conference on Communications, Jun 2013, Hungary. hal-00875051

\section{HAL Id: hal-00875051 https://hal.science/hal-00875051}

Submitted on 21 Oct 2013

HAL is a multi-disciplinary open access archive for the deposit and dissemination of scientific research documents, whether they are published or not. The documents may come from teaching and research institutions in France or abroad, or from public or private research centers.
L'archive ouverte pluridisciplinaire HAL, est destinée au dépôt et à la diffusion de documents scientifiques de niveau recherche, publiés ou non, émanant des établissements d'enseignement et de recherche français ou étrangers, des laboratoires publics ou privés. 


\title{
Gaussian Half-Duplex Relay Channels: Generalized Degrees of Freedom and Constant Gap Result
}

\author{
Martina Cardone ${ }^{\dagger}$, Daniela Tuninetti*, Raymond Knopp ${ }^{\dagger}$, Umer Salim ${ }^{\ddagger}$ \\ ${ }^{\dagger}$ Eurecom, Campus SophiaTech, 450 Route des Chappes, 06410 Biot Email: \{cardone, knopp\}@eurecom.fr \\ * University of Illinois at Chicago, Chicago, IL 60607, USA, Email: danielat@uic.edu \\ $\ddagger$ Intel Mobile Communications Sophia Antipolis, 06560, France, Email: umer.salim@intel.com
}

\begin{abstract}
This paper considers the Gaussian relay channel where the relay node operates in half-duplex mode. The exact capacity of the linear deterministic approximation of the Gaussian channel at high SNR is derived first. This result is then used to inspire an achievable scheme valid for any SNR in the original channel. The scheme is quite simple: it uses successive decoding and does not incur in the typical delay of backward decoding. The achievable rate is then showed to be at most 3 bits away from the cut-set upper bound, which allows to analytically determine the generalized Degrees-of-Freedom of the channel. A closed form expression for the gDoF-optimal fraction of time the relay node transmits is found as well.
\end{abstract}

\section{INTRODUCTION}

In wireless systems, cooperation among nodes that share the same resources results in an overall system performance enhancement. One of the simplest form of cooperation is represented by the Relay Channel (RC), where a source terminal communicates to a destination with the help of a relay node. The relay can primarily operate in two modes: FullDuplex (FD) and Half-Duplex (HD). In the former the relay simultaneously transmits and receives over the same communication resource; in the latter the relay can either transmit or receive at any given time, but not both. FD relaying in wireless networks has practical restrictions; the enormous difference in transmit and received powers, in fact, makes it infeasible to suppress self-interference. As a result HD relaying proves to be a more realistic and practical technology with its relatively simple signal processing. From an application point of view, the HD model fits future $4 \mathrm{G}$ network with relays [1].

Related Work. The RC has been widely studied in information theory but its capacity is still not known in general [2]. The RC was first introduced by van der Meulen [3] and then thoroughly studied by Cover and El Gamal [4]. In this pioneering work they found a max-flow min-cut outer bound, or cut-set for short, and developed two achievable relaying strategies: the Decode-and-Forward (DF) and the Compressand-Forward $(\mathrm{CF})$. In the $\mathrm{DF}$, the relay fully decodes the information coming from the source, then re-encodes it and sends it to the destination. On the other hand in the CF, the relay compresses the information received and then sends it to the destination. The combination of $\mathrm{CF}$ and $\mathrm{DF}$ is known to be the best achievable rate for the RC [4].

Recently, research on HD relaying has received considerable attention both from an information and communication point of view. In [5] authors investigated the Diversity Multiplexing
Tradeoff for the single HD RC and they proved that it meets the $2 \times 1$-MISO bound. In [6] both the FD and the HD RCs are analyzed; in particular the effect of correlated noises at the destination and at the relay is discussed. In [7], the authors studied multi-relay networks and demonstrated that QuantizeMap-and-Forward (QMF) scheme, i.e., a network extension of $\mathrm{CF}$, based on nested lattice codes achieves the capacity to within a constant gap; this gap, in the single-relay case, is of 5 bits as originally computed in [8] but where Gaussian codes were used. In [9], authors considered a network with multiple relays where the relays listen and transmit phases are fixed and known a priori to all nodes; here authors developed an iterative algorithm to find the optimal relay scheduling, i.e., the time fraction each relay either transmits or receives. In [10] we also studied the multi-relay network and proposed a scheme based on Noisy-Network-Coding (NNC), i.e., a network extension of $\mathrm{CF}$, and found a smaller gap than that in [7].

This work concentrates on the single HD relay case with the objective to develop a practical scheme, that is less complex than DF or variations of CF presented in the literature, and still achieves capacity to within a constant gap.

Contributions and Paper Organization. In this work we study the Gaussian HD RC, described in Section II, whose capacity is still unknown. In Section III, we first analyze the Linear Deterministic Approximation (LDA) of the Gaussian channel at high SNR in the spirit of [8] and characterize its capacity; we show that a random relay switch and non i.i.d. $\operatorname{Ber}(1 / 2)$ bits are optimal. We then move, in Section IV, to the Gaussian HD RC; we analytically determine its gDoF and show that the cut-set upper bound is achievable to within a constant gap. This is accomplished by developing a simple achievable scheme inspired by the LDA which is quite simple both in the encoding and decoding phases. We also derive the gDoF-wise optimal fraction of time the relay transmits in closed form. We remark here that in deriving the outer bound we do not develop a separate theory for memoryless networks with HD nodes, but we incorporate the HD constraints into the memoryless FD framework as in [11]. Section V concludes the paper. Some of the proofs can be found in the Appendix.

\section{SYSTEM MODEL}

In the following we use the notation convention of [2].

The General Memoryless FD RC. A general FD RC consists of two input alphabets $\left(\mathcal{X}_{s}, \mathcal{X}_{r}\right)$, two output alphabets 
$\left(\mathcal{Y}_{r}, \mathcal{Y}_{d}\right)$ and a memoryless channel with transition probability $P_{Y_{r}, Y_{d} \mid X_{s}, X_{r}}$. The source has a message $W$ uniformly distributed on $\left[1: 2^{N R}\right]$ for the destination, where $N$ denotes the codeword length and $R \geq 0$ the transmission rate measured in bits per channel use (i.e., logarithms are in base 2). At time $i, i \in[1: N]$, the source maps the message $W$ into the input symbol $X_{s, i}(W)$, and the relay maps its past channel observations into the input symbol $X_{r, i}\left(Y_{r}^{i-1}\right)$. At time $N$, the destination makes an estimate of $W$ based on all its channel observations as $\widehat{W}\left(Y_{d}^{N}\right)$. The capacity is the largest rate $R$ such that $\mathbb{P}[\widehat{W} \neq W] \rightarrow 0$ as $N \rightarrow+\infty$.

The capacity of the general memoryless FD RC is unknown. The best upper bound is the cut-set bound [2, Thm.16.1]

$R \leq r^{(\mathrm{CS})}:=\max _{P_{X_{s}, X_{r}}} \min \left\{I\left(X_{s}, X_{r} ; Y_{d}\right), I\left(X_{s} ; Y_{r}, Y_{d} \mid X_{r}\right)\right\}$.

The best inner bound is a combination of CF and DF [4].

The Gaussian HD RC. A HD RC is a special memoryless FD RC [11] where, with a slight abuse of notation compared to the previous paragraph, the channel input of the relay is the pair $\left(X_{r}, S_{r}\right)$, with $X_{r} \in \mathcal{X}_{r}$ and $S_{r} \in\{0,1\}$ representing the state random variable that indicates whether the relay is in receive-mode $\left(S_{r}=0\right)$ or in transmit-mode $\left(S_{r}=1\right)$. The single-antenna Gaussian HD RC has input/output relationship

$$
\begin{aligned}
& Y_{r}=\mathrm{h}_{\mathrm{sr}} X_{s}\left(1-S_{r}\right)+Z_{r}, \\
& Y_{d}=\mathrm{h}_{\mathrm{sd}} X_{s}+\mathrm{h}_{\mathrm{rd}} X_{r} S_{r}+Z_{d},
\end{aligned}
$$

where the channel gains $h_{s d}, h_{s r}, h_{r d}$ are complex-valued and constant, and the channel inputs are subject to the average power constraints $\mathbb{E}\left[\left|X_{i}\right|^{2}\right] \leq P_{i} \in \mathbb{R}^{+}, i \in\{s, r\}$. We assume without loss of generality that the noises are propercomplex Gaussian random variables with zero-mean and unit variance. We further assume that $Z_{d}$ is independent of $Z_{r}$. Note that the relay can convey at most 1 bit of information to the destination through the binary state $S_{r}$; in this case we say that the relay employs random switch between the receiveand transmit-phases [11]. On the other hand, if the receive- and transmit-phases are fixed and a priori known to all nodes, we say that the relay employs a deterministic switch.

The capacity of the channel in (1) is unknown. Here we make progress towards determining its capacity by first establishing its gDoF, i.e., an approximate capacity characterization at high-SNR [8], and then showing that the capacity outer bound is achievable to within a constant gap for any choice of the channel parameters. For a SNR $>0$ consider the parameterization

$$
\begin{aligned}
& P_{s}\left|\mathrm{~h}_{\mathrm{sd}}\right|^{2}=S=\mathrm{SNR}^{\beta_{\mathrm{sd}}}, \text { source-destination link } \\
& P_{r}\left|\mathrm{~h}_{\mathrm{rd}}\right|^{2}=C=\mathrm{SNR}^{\beta_{\mathrm{rd}}}, \text { relay-destination link } \\
& P_{s}\left|\mathrm{~h}_{\mathrm{sr}}\right|^{2}=I=\mathrm{SNR}^{\beta_{\mathrm{sr}}}, \text { source-relay link, }
\end{aligned}
$$

for some non-negative $\left(\beta_{\mathrm{sd}}, \beta_{\mathrm{rd}}, \beta_{\mathrm{sr}}\right)$. The $\mathrm{gDoF}$ is defined as

$$
\mathrm{d}:=\lim _{\mathrm{SNR} \rightarrow+\infty} \frac{\mathrm{C}(S, I, C)}{\log (1+\mathrm{SNR})},
$$

where $\mathrm{C}(S, I, C)=\max \{R\}$ is the capacity of the channel in (1). The gDoF characterizes the capacity in the limit for infinite SNR. At finite SNR the capacity is said to be known to within $\mathrm{b}$ bits if one can show rates $r^{(\mathrm{in})}$ and $r^{\text {(out) }}$ such that

$$
r^{(\text {in })} \leq \mathrm{C}(S, I, C) \leq r^{(\text {out })} \leq r^{(\text {in })}+\mathrm{b} \log (2) .
$$

To obtain insights into the behavior of the capacity of the Gaussian HD RC in (1), we consider first its LDA at high SNR [8]. The LDA is a noiseless HD RC which input/output relationship is

$$
\begin{aligned}
& Y_{r}=\mathbf{S}^{n-\beta_{\mathrm{sr}}} X_{s}\left(1-S_{r}\right), \\
& Y_{d}=\mathbf{S}^{n-\beta_{\mathrm{sd}}} X_{s}+\mathbf{S}^{n-\beta_{\mathrm{rd}}} X_{r} S_{r},
\end{aligned}
$$

where $\left(\beta_{\mathrm{sr}}, \beta_{\mathrm{sd}}, \beta_{\mathrm{rd}}\right)$ are integer numbers, $\left(X_{s}, X_{r}, Y_{r}, Y_{d}\right)$ are vectors of length $n:=\max \left\{\beta_{\mathrm{sr}}, \beta_{\mathrm{sd}}, \beta_{\mathrm{rd}}\right\}, \mathbf{S}$ is the $n \times n$ shift matrix [8], and $S_{r}$ is the state of the relay.

\section{THE CAPACITY OF THE LDA AND A SIMPLE ACHIEVABLE SCHEME (OPTIMAL TO WITHIN 1 BIT)}

In this section we characterize the capacity of the LDA in (3). It is well known that the capacity of a general noiseless $\mathrm{RC}$ is given by the cut-set bound $r^{(\mathrm{CS})}$ [2]. We first evaluate the cut-set bound for the LDA and show that the optimal input distribution has a random switch and does not correspond to i.i.d. Ber(1/2) bits. We then derive a simple achievable scheme (with deterministic switch and i.i.d. Ber(1/2) input bits) that is optimal to within 1 bit. This latter scheme motivates the asymptotically optimal scheme for the Gaussian HD RC presented in Section IV.

Theorem 1 The capacity of the LDA in (3) is given by

$\mathrm{C}^{(\mathrm{HD}-\mathrm{LDA})}=\beta_{\mathrm{sd}}+\max _{\gamma \in[0,1]} \min \left\{\left(1-\theta^{*}(\gamma)\right) \log \frac{1}{1-\theta^{*}(\gamma)}\right.$

$\left.+\theta^{*}(\gamma) \log \frac{L-1}{\theta^{*}(\gamma)}, \gamma\left[\beta_{\mathrm{sr}}-\beta_{\mathrm{sd}}\right]^{+}\right\}$for $\beta_{\mathrm{sr}}>\beta_{\mathrm{sd}}, \beta_{\mathrm{rd}}>\beta_{\mathrm{sd}}$,

$\mathrm{C}^{(\mathrm{HD}-\mathrm{LDA})}=\beta_{\mathrm{sd}} \quad$ for $\beta_{\mathrm{sr}} \leq \beta_{\mathrm{sd}}$ or $\beta_{\mathrm{rd}} \leq \beta_{\mathrm{sd}}$.

where $\theta^{*}(\gamma)=1-\max \left\{2^{-\left[\beta_{\mathrm{rd}}-\beta_{\mathrm{sd}}\right]^{+}}, \gamma\right\}$.

Proof: We distinguish two cases.

Case 1: $\beta_{\mathrm{rd}} \leq \beta_{\mathrm{sd}}$ or $\beta_{\mathrm{sr}} \leq \beta_{\mathrm{sd}}$. In general the capacity of any HD RC is upper bounded by that of the corresponding FD RC. The capacity of the FD version of the LDA in (3) is

$$
\begin{aligned}
\mathrm{C}^{(\mathrm{FD}-\mathrm{LDA})} & =\max _{P_{X_{s}, X_{r}}} \min \left\{H\left(Y_{d}\right), H\left(Y_{r}, Y_{d} \mid X_{r}\right)\right\} \\
& =\beta_{\mathrm{sd}}+\min \left\{\left[\beta_{\mathrm{rd}}-\beta_{\mathrm{sd}}\right]^{+},\left[\beta_{\mathrm{sr}}-\beta_{\mathrm{sd}}\right]^{+}\right\},
\end{aligned}
$$

achieved by i.i.d. $\operatorname{Ber}(1 / 2)$ input bits. Hence, in the regime of interest $\mathrm{C}^{(\mathrm{FD}-\mathrm{LDA})}=\beta_{\mathrm{sd}}$. Since $\beta_{\mathrm{sd}}$ bits can be conveyed from the source to the destination by silencing the relay we conclude that $\mathrm{C}^{(\mathrm{HD}-\mathrm{LDA})}=\beta_{\mathrm{sd}}$ in this regime.

Case 2: $\beta_{\mathrm{rd}}>\beta_{\mathrm{sd}}$ and $\beta_{\mathrm{sr}}>\beta_{\mathrm{sd}}$. We start by writing $Y_{d}=\left[Y_{d, u}, Y_{d, l}\right]$, where: (i) $Y_{d, l}$ ("orange bits" labeled $b_{1}[2]$ in Fig 1(b)) contains the lower $\beta_{\text {sd }}$ bits of $Y_{d}$; these bits are 


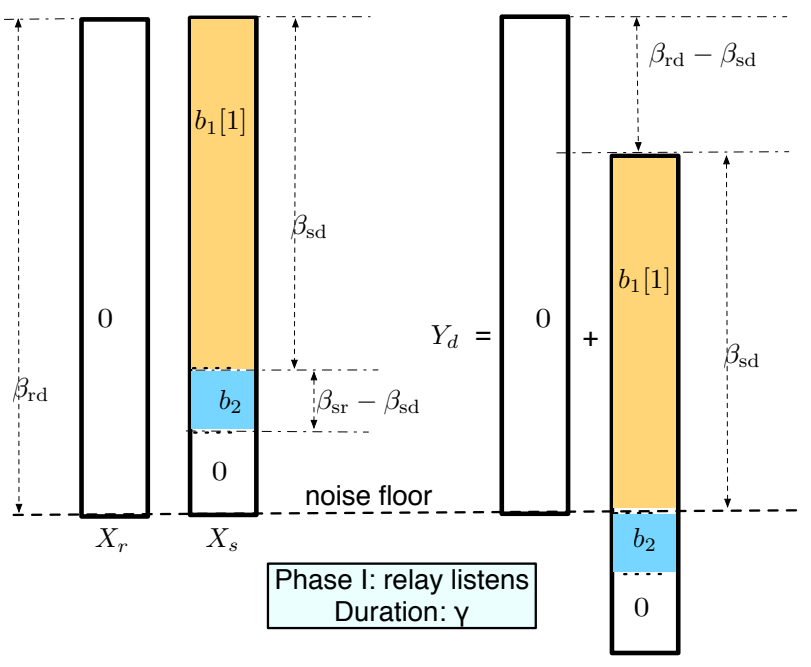

(a) Phase I: Relay receives.

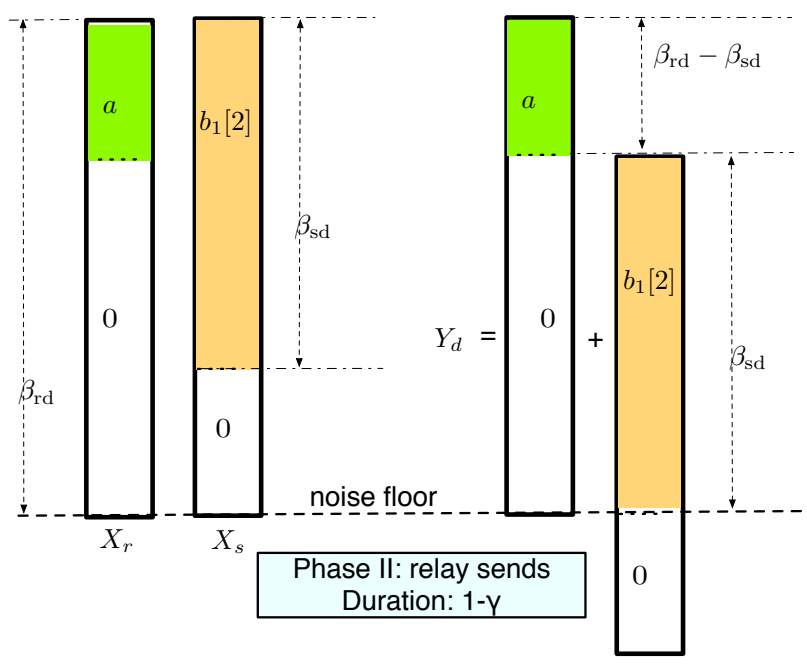

(b) Phase II: Relay transmits.

Fig. 1. A scheme for the LDA that is optimal to within one 1 bit.

a combination of the bits of $X_{s}$ and the lower bits of $X_{r}$, indicated as $X_{r, l}$; (ii) $Y_{d, u}$ ("green bits" labeled $a$ in Fig 1(b)) contains the upper $\beta_{\mathrm{rd}}-\beta_{\mathrm{sd}}$ bits of $Y_{d}$, which only depend on the upper bits of $X_{r}$, indicated as $X_{r, u}$. Thus we have

$$
H\left(Y_{d}\right) \leq H\left(Y_{d, u}\right)+H\left(Y_{d, l}\right) \leq H\left(Y_{d, u}\right)+\beta_{\mathrm{sd}},
$$

since $Y_{d, l}$ contains $\beta_{\mathrm{sd}}$ bits. Next the distribution of $Y_{d, u}$ is

$$
\mathbb{P}\left[Y_{d, u}=y\right]=\gamma \delta[y]+(1-\gamma) \mathbb{P}\left[X_{r, u}=y \mid S_{r}=1\right]
$$

for $y \in[0: L-1], L:=2^{\beta_{\text {rd }}-\beta_{\text {sd }}}>1$, where $\delta[y]=1$ if $y=0$ and zero otherwise, and where $\gamma:=\mathbb{P}\left[S_{r}=0\right]$ (i.e., the fraction of time the relay listens to the channel). Note that $Y_{d, u}$ is zero when either the relay is silent (with probability $\gamma)$ or the relay is active and sends the whole-zero vector (with probability $(1-\gamma))$. Then

$$
H\left(Y_{d, u}\right) \leq(1-\theta) \log \frac{1}{1-\theta}+\theta \log \frac{L-1}{\theta}
$$

where $\theta:=\mathbb{P}\left[Y_{d, u} \neq 0\right] \in[0,1-\gamma]$. The upper bound on $H\left(Y_{d, u}\right)$ is maximized by $\theta=\theta^{*}:=1-\max \{1 / L, \gamma\}$. Thus

$$
\begin{gathered}
\mathrm{C}^{(\mathrm{HD}-\mathrm{LDA})}=\max _{P_{X_{s}, X_{r}, S_{r}} \min \left\{H\left(Y_{d}\right), H\left(Y_{r}, Y_{d} \mid X_{r}, S_{r}\right)\right\}} \\
\leq \beta_{\mathrm{sd}}+\max _{\gamma \in[0,1]} \min \left\{\left(1-\theta^{*}\right) \log \frac{1}{1-\theta^{*}}+\theta^{*} \log \frac{L-1}{\theta^{*}},\right. \\
\left.\gamma\left[\beta_{\mathrm{sr}}-\beta_{\mathrm{sd}}\right]^{+}\right\},
\end{gathered}
$$

since $H\left(Y_{r}, Y_{d} \mid X_{r}, S_{r}\right) \leq \gamma \max \left\{\beta_{\mathrm{sr}}, \beta_{\mathrm{sd}}\right\}+(1-\gamma) \beta_{\mathrm{sr}}$.

The above upper bound on $\mathrm{C}^{(\mathrm{HD}-\mathrm{LDA})}$ is achievable. Let $S_{r} \sim \operatorname{Ber}(1-\gamma)$ independent of everything else, and $X_{s}$ and $X_{r}$ be independent. The source uses i.i.d. $\operatorname{Ber}(1 / 2)$ bits; the relay uses i.i.d. $\operatorname{Ber}(0)$ bits for $X_{r, l}$ and $\mathbb{P}\left[X_{r, u}=y \mid S_{r}=\right.$ 1] $=p_{0}^{*}$ if $y=0$ and $\mathbb{P}\left[X_{r, u}=y \mid S_{r}=1\right]=\left(1-p_{0}^{*}\right) /(L-$ 1) otherwise, for $p_{0}^{*}=\frac{[1 / L-\gamma]^{+}}{1-\gamma}$, for $X_{r, u}$; the distribution of $X_{r, u}$ in state $S_{r}=0$ is irrelevant since its contribution at the destination is zero anyway, so we can assume that the input distribution for $X_{r}$ is independent of the state $S_{r}$. With this choice, straightforward computations show that the upper bound on $\mathrm{C}^{(\mathrm{HD}-\mathrm{LDA})}$ is achievable thereby concluding the proof.

The capacity in Thm. 1 shows that i.i.d. Ber(1/2) input bits and random switch are not optimal. Indeed, with i.i.d. $\operatorname{Ber}(1 / 2)$ input bits and deterministic switch one can achieve

$$
\begin{aligned}
& r^{(\mathrm{HD}-\mathrm{LDA})}= \beta_{\mathrm{sd}}+\gamma^{(\mathrm{LDA})}\left[\beta_{\mathrm{sr}}-\beta_{\mathrm{sd}}\right]^{+} \text {since } \\
& r^{(\mathrm{HD}-\mathrm{LDA})}=\max _{P_{X_{s}, X_{r}, S_{r}} \min \left\{H\left(Y_{d} \mid S_{r}\right), H\left(Y_{r}, Y_{d} \mid X_{r}, S_{r}\right)\right\}} \\
&=\max _{\gamma \in[0,1]} \min \left\{\gamma \beta_{\mathrm{sd}}+(1-\gamma) \max \left\{\beta_{\mathrm{sd}}, \beta_{\mathrm{rd}}\right\},\right. \\
&\left.\gamma \max \left\{\beta_{\mathrm{sd}}, \beta_{\mathrm{sr}}\right\}+(1-\gamma) \beta_{\mathrm{sd}}\right\}
\end{aligned}
$$

and where the optimal $\gamma$ is

$$
\gamma^{(\mathrm{LDA})}= \begin{cases}\frac{\left(\beta_{\mathrm{rd}}-\beta_{\mathrm{sd}}\right)}{\left(\beta_{\mathrm{rd}}-\beta_{\mathrm{sd}}\right)+\left(\beta_{\mathrm{sr}}-\beta_{\mathrm{sd}}\right)} & \text { for } \beta_{\mathrm{rd}}>\beta_{\mathrm{sd}}, \beta_{\mathrm{sr}}>\beta_{\mathrm{sd}} \\ 0 & \text { otherwise. }\end{cases}
$$

Notice that $\mathrm{C}^{(\mathrm{HD}-\mathrm{LDA})}-r^{(\mathrm{HD}-\mathrm{LDA})} \leq H\left(S_{r}\right) \leq \log (2)$, that is, i.i.d. $\operatorname{Ber}(1 / 2)$ input bits and deterministic switch are optimal to within 1 bit. The scheme that achieves $r^{(\mathrm{HD}-\mathrm{LDA})}$ is shown in Figs. 1(a) and 1(b) for the case $\min \left\{\beta_{\mathrm{sr}}, \beta_{\mathrm{rd}}\right\}>$ $\beta_{\text {sd }}$. In Phase I/Fig. 1(a) the relay listens and the source sends $b_{1}$ (of length $\beta_{\text {sd }}$ bits) directly to the destination and $b_{2}$ (of length $\beta_{\mathrm{sr}}-\beta_{\mathrm{sd}}$ bits) to the relay; the duration of Phase I is $\gamma$, hence the relay has accumulated $\gamma\left(\beta_{\mathrm{sr}}-\beta_{\mathrm{sd}}\right)$ bits to forward to the destination. In Phase II/Fig. 1(b) the relay forwards the bits learnt in Phase I to the destination by 'repackaging' them into $a$ (of length $\beta_{\mathrm{rd}}-\beta_{\mathrm{sd}}$ bits); the source keeps sending a new $b_{1}$ (of length $\beta_{\mathrm{sr}}-\beta_{\mathrm{sd}}$ bits) directly to the destination; the duration of Phase II is such that all the bits accumulated in Phase I can be delivered to the destination, that is $\gamma\left(\beta_{\mathrm{sr}}-\right.$ $\left.\beta_{\mathrm{sd}}\right)=(1-\gamma)\left(\beta_{\mathrm{rd}}-\beta_{\mathrm{sd}}\right)$, which gives precisely the optimal $\gamma^{(\mathrm{LDA})}$. The total number of bits decoded at the destination is $1 \cdot \beta_{\mathrm{sd}}+\gamma^{(\mathrm{LDA})} \cdot\left(\beta_{\mathrm{sr}}-\beta_{\mathrm{sd}}\right)$, as claimed. 
IV. THE GDOF AND THE CAPACITY TO WITHIN 3 BITS FOR THE GAUSSIAN HD RC

In this section we shall mimic the strategy in Figs. 1(a) and 1(b) for the HD Gaussian RC. With this we can show:

Theorem 2 The cut-set upper bound for the HD Gaussian RC is achievable to within 3 bits. This characterizes the $g D o F$ as

$$
\mathrm{d}=\beta_{\mathrm{sd}}+\gamma^{(\mathrm{LDA})}\left[\beta_{\mathrm{sr}}-\beta_{\mathrm{sd}}\right]^{+} .
$$

Proof: Upper Bound. The capacity of the HD Gaussian $\mathrm{RC}$ is upper bounded by

$$
\begin{aligned}
& \mathrm{C}(S, I, C) \leq r^{(\mathrm{CS}-\mathrm{HD}-\mathrm{G})}:=\max _{\left(\gamma, P_{s, 0}, P_{s, 1}, P_{r, 0}, P_{r, 1}\right) \in \mathbb{R}_{+}^{5}} \\
& \quad \min \left\{H\left(S_{r}\right)+\gamma I_{1}+(1-\gamma) I_{2}, \gamma I_{3}+(1-\gamma) I_{4}\right\},
\end{aligned}
$$

where

$$
\begin{aligned}
& I_{1}:=\log \left(1+\left|\mathrm{h}_{\mathrm{sd}}\right|^{2} P_{s, 0}\right), \\
& I_{2}:=\log \left(1+\left(\sqrt{\left|\mathrm{h}_{\mathrm{sd}}\right|^{2} P_{s, 1}}+\sqrt{\left|\mathrm{h}_{\mathrm{rd}}\right|^{2} P_{r, 1}}\right)^{2}\right) \\
& I_{3}:=\log \left(1+\left(\left|\mathrm{h}_{\mathrm{sr}}\right|^{2}+\left|\mathrm{h}_{\mathrm{sd}}\right|^{2}\right) P_{s, 0}\right), \\
& I_{4}:=\log \left(1+\left|\mathrm{h}_{\mathrm{sd}}\right|^{2} P_{s, 1}\right),
\end{aligned}
$$

with $\gamma P_{u, 0}+(1-\gamma) P_{u, 1} \leq P_{u}, u \in\{s, r\}$. This upper bound follows from the cut-set bound $r^{(\mathrm{CS})}$ by using

$$
I\left(S_{r} ; Y_{d}\right) \leq H\left(S_{r}\right)=-\gamma \log (\gamma)-(1-\gamma) \log (1-\gamma),
$$

and by the fact that in state $S_{r}=\ell \in[0: 1]$ joint Gaussian inputs with powers $\left(P_{s, \ell}, P_{r, \ell}\right)$, such that the average power constraints are satisfied, maximize the differential entropies. The upper bound $r^{(\mathrm{CS}-\mathrm{HD}-\mathrm{G})}$ implies that (4) is a $\mathrm{gDoF}$ upper bound (the details of the proof are in Appendix).

Lower Bound. We can mimic the strategy developed for the LDA as follows. We assume $S \leq C$, otherwise we use direct transmission to achieve $R=\log (1+S)$. The transmission is divided into two phases. In Phase I of duration $\gamma$ the transmit signals are

$$
X_{s}[1]=\sqrt{1-\delta} X_{b_{1}[1]}+\sqrt{\delta} X_{b_{2}}, X_{r}[1]=0, \delta:=\frac{1}{1+S} .
$$

The relay applies successive decoding of $X_{b_{1}[1]}$ followed by $X_{b_{2}}$ from $Y_{r}[1]=\sqrt{C} \sqrt{1-\delta} X_{b_{1}[1]}+\sqrt{C} \sqrt{\delta} X_{b_{2}}+Z_{r}[1]$, which is possible if

$$
\begin{aligned}
R_{b_{1}[1]} & \leq \gamma \log (1+C)-\gamma \log (1+C \delta) \\
R_{b_{2}} & \leq \gamma \log (1+C \delta) .
\end{aligned}
$$

The destination decodes $X_{b_{1}[1]}$ treating $X_{b_{2}}$ as noise from $Y_{d}[1]=\sqrt{S} \sqrt{1-\delta} X_{b_{1}[1]}+\sqrt{S} \sqrt{\delta} X_{b_{2}}+Z_{d}[1]$, which is possible if

$$
R_{b_{1}[1]} \leq \gamma \log (1+S)-\gamma \log (1+S \delta) .
$$

Finally, since we assume $S \leq C$, Phase I is successful if (6) and (7) are satisfied. In Phase II of duration $1-\gamma$ the transmit signals are

$$
X_{s}[2]=X_{b_{1}[2]}, \quad X_{r}[2]=X_{b_{2}} .
$$

The destination applies successive decoding of $X_{b_{2}}$ followed by $X_{b_{1}[2]}$ from $Y_{d}[2]=\sqrt{S} X_{b_{1}[2]}+\sqrt{I} X_{b_{2}}+Z_{d}[2]$, which is possible if

$$
\begin{aligned}
R_{b_{2}} & \leq(1-\gamma) \log (1+I \delta) \\
R_{b_{1}[2]} & \leq(1-\gamma) \log (1+S) .
\end{aligned}
$$

By imposing that the rate $R_{b_{2}}$ is the same in both phases, that is, that (6) and (8) are equal, we get that $\gamma$ should be chosen equal to

$$
\gamma^{*}=\frac{\log \left(1+\frac{I}{1+S}\right)}{\log \left(1+\frac{I}{1+S}\right)+\log \left(1+\frac{C}{1+S}\right)} .
$$

Note: $\lim _{\mathrm{SNR} \rightarrow \infty} \gamma^{*}=\gamma^{(\mathrm{LDA})}$. The rate sent directly from the relay to the destination, that is, the sum of (7) and (9), is

$$
R_{b_{1}[1]}+R_{b_{1}[2]}=\log (1+S)-\gamma^{*} \log \left(1+\frac{S}{1+S}\right),
$$

therefore the total rate in the two phases is

$$
\begin{aligned}
& \mathrm{C}(S, I, C) \geq R_{b_{1}[1]}+R_{b_{1}[2]}+R_{b_{2}} \geq r^{(\mathrm{HD}-\mathrm{G})} \\
& :=\log (1+S)\left(1+\frac{\left(c_{1}-1\right)\left(c_{2}-1\right)}{\left(c_{1}-1\right)+\left(c_{2}-1\right)}\right),
\end{aligned}
$$

where

$$
\begin{aligned}
& c_{1}:=\frac{\log (1+I+S)}{\log (1+S)} \geq 1 \\
& c_{2}:=\frac{\log (1+C+S)}{\log (1+S)} \geq 1 .
\end{aligned}
$$

Note: the above rate is completely symmetric with respect $C$ and $I$. By similar steps as done in the Appendix, one can show that $r^{(\mathrm{HD}-\mathrm{G})}$ in (10) implies that (4) is achievable.

Gap. We upper bound $r^{(\mathrm{CS}-\mathrm{HD}-\mathrm{G})}-r^{(\mathrm{HD}-\mathrm{G})}$.

Case 1: $S \leq C$. The gap is given by

$$
\mathrm{GAP} \leq 2 \log (2)+\frac{x^{2}+y^{2}+x y+x}{x^{2}+y^{2}+2 x y+x+y} \leq 3 \mathrm{bits},
$$

where $x:=\log \left(1+\frac{I}{1+S}\right)$ and $y:=\log \left(1+\frac{C}{1+S}\right)$.

Case 2: $S>C$. In this case the relay stays silent and the achievable rate is $R=\log (1+S)$. The gap is

$$
\begin{aligned}
& \text { GAP } \leq 2 \log (2)+\log (1+S) \frac{\left(b_{1}-1\right)\left(b_{2}-1\right)}{\left(b_{1}-1\right)+\left(b_{2}-1\right)} \\
& \leq 2 \log (2)+\log (1+S)\left(b_{2}-1\right) \\
& =2 \log (2)+\log \left(1+\frac{C}{1+S}\right) \leq 3 \text { bits, }
\end{aligned}
$$

where the last inequality is due to the fact that $C<S$.

We conclude by pointing out some important practical aspects of our LDA-inspired achievable scheme: (a) the destination does not use backward decoding, which simplifies the decoding procedure and incurs no delay, (b) the destination uses successive decoding, which is simpler than joint decoding, and (c) no power allocation has been applied at the relay or at the source, which simplifies the encoding procedure. These observations point to an interesting practical tradeoff 
between complexity and performance: our simple scheme is at most 3 bits far from the capacity but a more complex (both in the encoding and decoding phases) scheme based on PartialDF (PDF) is at most 1 bit from the capacity [12]. However, the gap found here is smaller than the 5 bits gap found in previous works based on CF-like schemes [8], [7]. In [12], we succeeded to further reduce this gap to 1.61 bits by using CF. In other words, more involved techniques (PDF and CF) are found to match the capacity to within a smaller gap at the cost of more complexity both in the encoding and decoding phases.

\section{CONCLUSIONS}

In this work we considered the half duplex relay channel. First we derived the exact capacity of the linear deterministic approximation of the Gaussian noise channel at high SNR; we showed that random switch is optimal and characterized the optimal input distribution; we also proposed a simple scheme that is optimal to within 1 bit. This simple scheme inspired an achievable scheme for the Gaussian noise channel at any SNR which we showed to be optimal to within 3 bits. Interestingly our simple scheme does not use power allocation or joint backward decoding. This leads to a tradeoff between complexity and performance.

\section{ACKNOWLEDGMENT}

The work of Dr. D. Tuninetti was partially funded by NSF under award number 0643954 and by Telecom-ParisTech, Paris France, while the author was on a sabbatical leave at the same institution. Eurecom's research is partially supported by its industrial partners: BMW Group Research \& Technology, IABG, Monaco Telecom, Orange, SAP, SFR, ST Microelectronics, Swisscom and Symantec. The research at Eurecom leading to these results has received funding from the EU FP7 grant agreement CONECT $n^{\circ}$ 257616. The research at IMC leading to these results has received funding from the EU FP7 grants SACRA ( $\left.{ }^{\circ} 249060\right)$ and iJOIN $\left(n^{\circ} 317941\right)$.

\section{APPENDIX}

Since the source transmits in both phases we let

$$
P_{s, 0}=\frac{\beta P_{s}}{\gamma}, \quad P_{s, 1}=\frac{(1-\beta) P_{s}}{1-\gamma}, \quad \beta \in[0,1] .
$$

On the other hand, the relay transmits only $(1-\gamma)$ the time and so in this phase it exploits all its available power, so

$$
P_{r, 0}=0, \quad P_{r, 1}=\frac{P_{r}}{1-\gamma} .
$$

Based on the CS outer bound given in (5) we have

$$
\begin{gathered}
r^{(\mathrm{CS}-\mathrm{HD}-\mathrm{G})} \leq \max _{\gamma \in[0,1]} \min \left\{\mathcal{H}(\gamma)+\gamma \log \left(1+\frac{S}{\gamma}\right)+\right. \\
+(1-\gamma) \log \left(1+\left(\sqrt{\frac{I}{1-\gamma}}+\sqrt{\frac{S}{1-\gamma}}\right)^{2}\right), \\
\left.\gamma \log \left(1+\frac{C}{\gamma}+\frac{S}{\gamma}\right)+(1-\gamma) \log \left(1+\frac{S}{1-\gamma}\right)\right\}
\end{gathered}
$$

$$
\begin{aligned}
= & \max _{\gamma \in[0,1]} \min \{2 \mathcal{H}(\gamma)+\gamma \log (\gamma+S)+ \\
& +(1-\gamma) \log \left(1-\gamma+(\sqrt{I}+\sqrt{S})^{2}\right), \\
& \mathcal{H}(\gamma)+\gamma \log (\gamma+C+S)+(1-\gamma) \log (1-\gamma+S)\} \\
\leq & 2+\log (1+S) \max _{\gamma \in[0,1]} \min \left\{\gamma+(1-\gamma) b_{1}, \gamma b_{2}+(1-\gamma)\right\} \\
= & 2+\log (1+S)\left(1+\frac{\left(b_{1}-1\right)\left(b_{2}-1\right)}{\left(b_{1}-1\right)+\left(b_{2}-1\right)}\right),
\end{aligned}
$$

where

$$
\begin{aligned}
& b_{1}:=\frac{\log \left(1+(\sqrt{I}+\sqrt{S})^{2}\right)}{\log (1+S)} \geq 1, \\
& b_{2}:=\frac{\log (1+C+S)}{\log (1+S)} \geq 1,
\end{aligned}
$$

and $\mathcal{H}(\gamma)=H\left(S_{r}\right)=-\gamma \log (\gamma)-(1-\gamma) \log (1-\gamma)$. The optimal $\gamma=\frac{\left(b_{1}-1\right)}{\left(b_{1}-1\right)+\left(b_{2}-1\right)} \in[0,1]$ is found by equating the two arguments of the max min (this is so because $\gamma+(1-\gamma) b_{1}$ is decreasing in $\gamma$ while $\gamma b_{2}+(1-\gamma)$ is increasing in $\left.\gamma\right)$. The rate above implies

$$
\begin{aligned}
\mathrm{d} & \leq \lim _{\mathrm{SNR} \rightarrow+\infty} \frac{\log (1+S)}{\log (1+\mathrm{SNR})}\left(1+\frac{\left(b_{1}-1\right)\left(b_{2}-1\right)}{\left(b_{1}-1\right)+\left(b_{2}-1\right)}\right) \\
& =\beta_{\mathrm{sd}}+\frac{\left[\beta_{\mathrm{rd}}-\beta_{\mathrm{sd}}\right]^{+}\left[\beta_{\mathrm{sr}}-\beta_{\mathrm{sd}}\right]^{+}}{\left[\beta_{\mathrm{rd}}-\beta_{\mathrm{sd}}\right]^{+}+\left[\beta_{\mathrm{sr}}-\beta_{\mathrm{sd}}\right]^{+}}
\end{aligned}
$$

which is equivalent to (4) after straightforward manipulations.

\section{REFERENCES}

[1] LTE-A, 3rd Generation Partnership Project; Technical Specification Group Radio Access Network; Evolved Universal Terrestrial Radio Access (EUTRA), 3GPP TR 36.806 V9.0.0, 2010.

[2] A. El Gamal and Y.H. Kim, Network Information Theory, Cambridge Univ. Press, Cambridge U.K., 2011.

[3] E. C. van der Meulen, "Three-terminal communication channel," Adv. Appl. Probab., vol. 3, pp. 120-154, 1971.

[4] T. Cover and A. El Gamal, "Capacity theorems for the relay channel," IEEE Trans. on Info. Theory, vol. 25, no. 5, pp. 572 - 584, Sept. 1979.

[5] S. Pawar, A.S. Avestimehr, and D.N.C. Tse, "Diversity-multiplexing tradeoff of the half-duplex relay channel," in 46th Annual Allerton Conf. on Commun., Control, and Computing, Sept. 2008, pp. 27 -33.

[6] L. Zhang, J. Jiang, A.J. Goldsmith, and S. Cui, "Study of gaussian relay channels with correlated noises," IEEE Trans. on Communication, vol. 59, no. 3, pp. 863 -876, March 2011.

[7] A. Özgür and S.N. Diggavi, "Approximately achieving gaussian relay network capacity with lattice codes," arxiv:1005.1284, 2010.

[8] A.S. Avestimehr, Wireless network information flow: a deterministic approach, Ph.D. thesis, EECS Department, University of California, Berkeley, Oct 2008.

[9] L. Ong, M. Motani, and S. J. Johnson, "On capacity and optimal scheduling for the half-duplex multiple-relay channel," IEEE Trans. on Info. Theory, vol. 58, no. 9, pp. 5770 -5784, Sept. 2012.

[10] M. Cardone, D. Tuninetti, R. Knopp, and U. Salim, "The capacity of the gaussian half-duplex multiple-relay network to within a constant gap," submitted to ISIT 2013, July 2013.

[11] G. Kramer, "Models and theory for relay channels with receive constraints," in 42nd Annual Allerton Conf. on Commun., Control, and Computing, Sept. 2004, pp. 1312-1321.

[12] M. Cardone, D. Tuninetti, R. Knopp, and U. Salim, "The capacity to within a constant gap of the gaussian half-duplex relay channel," submitted to ISIT 2013, July 2013. 